OPEN ACCESS

Edited by:

Sunil Khare,

Indian Institute of Technology Delhi,

India

Reviewed by:

Rani Gupta,

University of Delhi, India

Likui Zhang,

Yangzhou University, China

${ }^{*}$ Correspondence:

Zunxi Huang

huangzunxi@163.com

Nanyu Han

ha0001yu@e.ntu.edu.sg

†These authors have contributed equally to this work

Specialty section: This article was submitted to

Extreme Microbiology,

a section of the journal

Frontiers in Microbiology

Received: 20 October 2019

Accepted: 17 February 2020

Published: 03 March 2020

Citation:

Jiang Z, Zhang $C$, Tang $M, X u B$, Wang L, Qian W, He J, Zhao Z, Wu Q, Mu Y, Ding J, Zhang $R$, Huang $Z$ and Han N (2020) Improving

the Thermostability of Rhizopus

chinensis Lipase Through

Site-Directed Mutagenesis Based on

B-Factor Analysis.

Front. Microbiol. 11:346.

doi: 10.3389/fmicb.2020.00346

\section{Improving the Thermostability of Rhizopus chinensis Lipase Through Site-Directed Mutagenesis Based on B-Factor Analysis}

\author{
Zhanbao Jiang ${ }^{1 t}$, Chengbo Zhang ${ }^{2 t}$, Minyuan Tang ${ }^{1}$, Bo X $u^{1,2,3,4}$, Lili Wang ${ }^{5}$, Wen Qian ${ }^{5}$, \\ Jiandong $\mathrm{He}^{5}$, Zhihong Zhao ${ }^{5}$, Qian $\mathrm{Wu}^{1,2,3,4}$, Yuelin $\mathrm{Mu}^{1,2,3,4}$, Junmei Ding ${ }^{1,2,3,4}$, \\ Rui Zhang 1,2,3,4, Zunxi Huang ${ }^{1,2,3,4 *}$ and Nanyu Han ${ }^{1,2,3,4 *}$ \\ ${ }^{1}$ School of Life Sciences, Yunnan Normal University, Kunming, China, ${ }^{2}$ Engineering Research Center of Sustainable \\ Development and Utilization of Biomass Energy, Ministry of Education, Yunnan Normal University, Kunming, China, ${ }^{3}$ Key \\ Laboratory of Yunnan for Biomass Energy and Biotechnology of Environment, Yunnan Normal University, Kunming, China, \\ ${ }^{4}$ Key Laboratory of Enzyme Engineering, Yunnan Normal University, Kunming, China, ${ }^{5}$ Yunnan Walvax Biotechnology Co., \\ Ltd., Kunming, China
}

In order to improve the thermostability of lipases derived from Rhizopus chinensis, we identified lipase (Lipr27RCL) mutagenesis sites that were associated with enhanced flexibility based upon B-factor analysis and multiple sequence alignment. We found that two mutated isoforms (Lipr27RCL-K64N and Lipr27RCL-K68T) exhibited enhanced thermostability and improved residual activity, with respective thermal activity retention values of $37.88 \%$ and $48.20 \%$ following a $2 \mathrm{~h}$ treatment at $50^{\circ} \mathrm{C}$ relative to wild type Lipr27RCL. In addition, these Lipr27RCL-K64N and Lipr27RCL-K68T isoforms exhibited 2.4- and 3.0-fold increases in enzymatic half-life following a 90 min incubation at $60^{\circ} \mathrm{C}$. Together these results indicate that novel mutant lipases with enhanced thermostability useful for industrial applications can be predicted based upon B-factor analysis and constructed via site-directed mutagenesis.

Keywords: lipase, thermostability, B-factor analysis, multiple sequence alignment, site-directed mutagenesis

\section{INTRODUCTION}

Lipases (triacylglycerol acyl hydrolases, EC 3. 1. 1.3) mediate the hydrolysis of triglycerides into monoglycerides, fatty acids, diglycerides, and glycerol at oil-water interfaces (Naik et al., 2010), and can further catalyze interesterification, esterification, alcoholysis, acidolysis, and aminolysis in non-aqueous environments (Pandey et al., 1999; Yu et al., 2014). Owing to their unique catalytic properties, lipases are utilized in a wide range of industrial contexts, such as in the production of food, leather, pharmaceuticals, and bioenergy (Persson et al., 2002; Ferreira-Dias et al., 2013).

Lipases are present in all forms of life, from microbes to mammals. Microbe-derived lipases exist in a wide variety of forms (Gupta et al., 2015). Compared with lipases from animals or plants, microbe-derived lipases can operate across a wider range of temperatures and $\mathrm{pH}$ values (Liu et al., 2017). Rhizopus-derived lipases have been shown to exhibit superior stability in acidic conditions, making them ideal for industrial applications. Dozens of commercial Rhizopus lipases have been produced to date, with Rhizopus chinensis CCTCC M201021 being the most commonly used strain for lipase production (Sha et al., 2013). However, lipases derived from R. chinensis are most active at 
moderate temperatures, whereas the reactions catalyzed by lipases in the context of oil processing require higher temperatures $\left(70-90^{\circ} \mathrm{C}\right)$ that can lead to the deactivation of lipases lacking heat resistance. As such, it is vital that heatresistant lipases should be developed so that they may be utilized as superior biocatalysts (Yu et al., 2012a,b).

Many different approaches have been employed to improve lipase kinetic and thermodynamic stability (Bommarius and Paye, 2013; Stepankova et al., 2013; Su et al., 2014). Directed evolution and semi-rational design are the two most common protein engineering approaches used to generate thermostable mutants of target enzymes (Li et al., 2018). Directed evolution through error-prone PCR and DNA shuffling is an effective means of improving the performance of enzymes under hightemperature conditions (Suen et al., 2004; Goomber et al., 2016). Screening through the many colonies necessary to identify superior enzyme isoforms, however, is time-consuming and cannot be effectively performed in host species that grow slowly. An alternative approach to improving enzymatic thermal stability that has been implemented successfully is the B-factor (Xie et al., 2014; Han et al., 2017). This approach relies upon improving thermostability via increasing enzymatic rigidity at certain sites, with B-factors derived from X-ray data offering insight into atom fluctuations and rigidity relative to their equilibrium positions (Ringe and Petsko, 1986). In the present study, we normalized the B-factor values of the Lipr27RCL X-ray structure and thereby identified residues with pronounced flexibility. Following multiple sequence alignment, we then conducted site-directed mutagenesis to improve $R$. chinensis lipase thermostability.

\section{MATERIALS AND METHODS}

\section{Materials}

Pichia pastoris GS115 was from Invitrogen (Shanghai, China). A site-directed mutagenesis kit and DMT chemically competent cells were from TransGen (Beijing, China). The R. chinensis lipr27RCL gene cloned in the pPIC9K vector was deposited in our laboratory. We obtained 4-nitrophenol palmitate (4-NPP) from Sigma. Mutagenic primers were synthesized by Shuoqing (Kunming, China). All other chemicals were commercially available analytical grade materials.

\section{Gene Cloning and Site-Directed Mutagenesis}

For site-directed mutagenesis experiments, we utilized pPIC9Kr27RCL as a template to construct four single-mutant lipase constructs (Lipr27RCL-K64N, Lipr27RCL-W65I, Lipr27RCLD66T, and Lipr27RCL-K68T) via introduction of point mutations into lipr27RCL through site-directed mutagenesis with a Fast MultiSite Mutagenesis System based on provided directions. The primers for these mutations are compiled in Table 1. We transformed the resultant PCR products into P. pastoris, and DNA sequencing was used to confirm the identity of the resulting recombinant strains. PCR thermocycler settings were as follows: $5 \mathrm{~min}$ at $94^{\circ} \mathrm{C}$, then 28 cycles of $30 \mathrm{~s}$ at $94^{\circ} \mathrm{C}, 2.5 \mathrm{~min}$ at $55^{\circ} \mathrm{C}$, and 2 min at $72^{\circ} \mathrm{C}$.
TABLE 1 | Oligonucleotide primers for Lipr27RCL-K64N, Lipr27RCL-W65I, Lipr27RCL-D66T, and Lipr27RCL-K68T.

\begin{tabular}{ll}
\hline Primers & Primer sequences \\
\hline r27RCL-K64N F & 5'-GTCGTTCCAGGTACCAATTGGGACTGTAAG-3' \\
r27RCL-K64N R & 5'-GAACATACTTGAGACATTGCTTACAGTCCCAA-3' \\
r27RCL-W65I F & 5'-GTTCCAGGTACCAAGATTGACTGGGACTGT-3' \\
r27RCL-W65I R & 5'-AGGAACATACTTGAGACATTGCTTACAGTCAAT-3' \\
r27RCL-D66T F & 5'-ACCAAGTTGGACTGTACGCAATGTCTCAAG-3' \\
r27RCL-D66T R & 5'-CTTACCATCAGGAACATACTTGAGACATTGCG-3' \\
r27RCL-K68T F & 5'-ACCAAGTGGGACTGTACGCAATGTCTCAAG-3' \\
r27RCL-K68T R & 5'-CTTACCATCAGGAACATACTTGAGACATTGCG-3'
\end{tabular}

\section{Protein Expression and Purification}

SalI was used to linearize the WT pPIC9K/Lipr27RCL and mutant constructs, which were then individually transformed into $P$. pastoris GS115 via electroporation. Yeast extract peptone dextrose (YPD) medium supplemented with $200 \mu \mathrm{g} / \mathrm{mL}$ G-418 (Geneticin) was used to select for clones, which were then grown for 2 days at $30^{\circ} \mathrm{C}$ in a $50 \mathrm{~mL}$ volume of buffered glycerolcomplex media. This media was then exchanged for $50 \mathrm{~mL}$ of buffered methanol-complex media to drive expression of the lipase proteins. Protein purification was conducted using an Amicon centrifugal filter device (cutoff 10.000). Proteins were then sequentially purified via $Q$ Sepharose ${ }^{\mathrm{TM}}$ Fast Flow anion exchange column chromatography and Phenyl-Sepharose 4 FF hydrophobic chromatography column chromatography, after which SDS-PAGE was used to confirm purification results (Figure 1 and Supplementary Figure S3), and a protein quantification kit was used to measure enzyme levels.

\section{Assessment of Enzymatic Activity}

We defined one unit of enzymatic activity $\left(\begin{array}{ll}1 & \mathrm{U}\end{array}\right)$ as the quantity of enzyme necessary to mediate the release of $1 \mu \mathrm{mol}$ p-nitrophenol (p-NP) per minute from 4-NPP (Li et al., 2011). Thermostability was analyzed through assessment of the residual enzymatic activity following incubation at $50^{\circ} \mathrm{C}$ for $5,10,15$, $20,30,40,50,65,80,100$, and $120 \mathrm{~min}$, and at $60^{\circ} \mathrm{C}$ for 1 , $3,6,10,15,20,25,30,35,45,60,75$, and $90 \mathrm{~min}$ at the established optimal $\mathrm{pH}$.

To establish key kinetic parameters for these purified lipases $\left(K_{m}, V_{\text {max }}\right.$, and $\left.k_{\text {cat }}\right)$, enzymes were measured in a Tris- $\mathrm{HCl}(\mathrm{pH}$ 9) buffer solution at $37^{\circ} \mathrm{C}$ with $4-\mathrm{PNN}$ as a substrate provided across a range of concentrations (0.078125-10 mM), with reactions monitored based on $\mathrm{p}-\mathrm{NP}$ production. LineweaverBurk plots were then used to fit the results and to determine these kinetic parameter values (Lineweaver and Burk, 1934).

\section{Lipase r27RCL Temperature Factor Calculation}

B-factor values for all Lipr27RCL C $\alpha$ atoms were extracted from the PDB file, these B-factors were normalized such that they had a zero mean and unit variance distribution as follows: $\mathrm{B}^{\prime}=\frac{\mathrm{B}-\langle\mathrm{B}\rangle}{\sigma(\mathrm{B})}$, where the $\langle\mathrm{b}\rangle$ is the average of all $\mathrm{C} \alpha$ atoms and $\sigma(\mathrm{B})$ is the standard deviation of the 


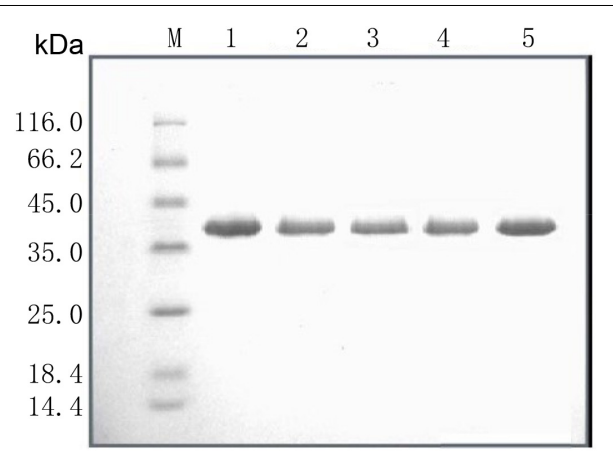

FIGURE 1 | SDS-PAGE analysis of the recombinant lipases. M: standard protein molecular mass markers; Lanes 1-5: Lipr27RCL, Lipr27RCL-K64N, Lipr27RCL-W65, Lipr27RCL-D66T, and Lipr27RCL-K68T, respectively.

B-factors for the individual protein (Yuan et al., 2003). We have successfully used these equations in previous studies (Han et al., 2017).

\section{Circular Dichroism (CD)}

Circular dichroism spectra were recorded using a Circular Dichroism (Model: Chirascan, Instrument: 0547) from Applied Photophysics Limited (United Kingdom). All spectra were recorded at $20^{\circ} \mathrm{C}$. Conditions, including pathlength: $1 \mathrm{~mm}$, time-per-point: $1 \mathrm{~s}(25 \mathrm{us} \times 40000)$, step size: $1 \mathrm{~nm}$, and bandwidth: $1 \mathrm{~nm}$, were used for scanning the 200-250 nm spectra range. CD spectra were collected from the solution of $50 \mathrm{mM}$ sodium phosphate buffer (PBS, $\mathrm{pH}$ 7.4) with the lipase concentration of $0.1 \mathrm{mg} / \mathrm{ml}$. After three scans, the final spectrum was corrected by removing the recorded baseline of the PBS control medium.

\section{Simulation Details}

The X-ray crystal structure of Lipr27RCL was taken from PDB 6A0W, while structures for the four recombinant lipases Lipr27RCL-K64N, Lipr27RCL-W65I, Lipr27RCLD66T, and Lipr27RCL-K68T, were constructed using the SWISS-MODEL server (Arnold et al., 2006), using the default parameters. Normal MD simulations of Lipr27RCL, Lipr27RCL-K64N, and Lipr27RCL-K68T were performed at $60^{\circ} \mathrm{C}$. After 1000 -step energy minimization, all systems were first equilibrated for $5 \mathrm{~ns}$ in NVT ensemble and then equilibrated for $5 \mathrm{~ns}$ in NPT ensemble by restraining all heavy atoms, and each system was simulated for 30 ns. All systems were solvated with TIP3P waters in an octahedral box, and the minimal distance between each protein and edge of the box was set to $1.0 \mathrm{~nm}$ (Jorgensen et al., 1983). Sodium and chloride ions were added with a concentration of $100 \mathrm{nM}$ to neutralize the systems. The GROMACS program suite version 4.5.7 and Amber ff99SB force field were applied in all simulations (Hornak et al., 2006; Hess et al., 2008). All simulations were performed in an isothermal-isobaric ensemble $\left(60^{\circ} \mathrm{C}, 1\right.$ bar $)$.

\section{RESULTS}

\section{Prediction of Mutagenesis Sites Based on B-Factor Analysis}

There is a linear relationship between B-factors determined based upon X-ray diffraction data and the mean square displacement of atoms relative to their average positions. As such, B-factors derived from protein crystal structures offer invaluable insights into the flexibility, stability, and dynamics of individual proteins. In the present study, we extracted and normalized the B-factor values of $\mathrm{C} \alpha$ atoms for Lipr27RCL from its crystal structure (Figure 2A). Residues from T63 to K68 (63-TKWDCK-68) of Lipr27RCL exhibited a high degree of flexibility. Multiple sequence alignment was then performed using 92 lipase sequences derived from thermophilic fungal lipases in the NCBI database, and beneficial sequences from residues 63 to 68 (63-TNITCT-68) were thereby discovered (Figure 2B). Based on the results from this normalized B-factor analysis and multi-sequence alignment, we thus identified $\mathrm{K} 64 \mathrm{~N}$, W65I, D66T, and K68T as putative sites that may improve Lipr27RCL thermostability.

\section{Construction and Characterization of Mutant Lipr27RCL Isoforms}

Based on the results from B-factor analysis and multiple sequence alignment, we used site-directed mutagenesis to produce mutant forms of Lipr27RCL in which the $64 \mathrm{~K}, 65 \mathrm{~W}, 66 \mathrm{D}$, and $68 \mathrm{~K}$ residues had been mutated to $64 \mathrm{~N}, 65 \mathrm{I}, 66 \mathrm{~T}$, and $68 \mathrm{~T}$, respectively. The resultant single mutant lipases (K64N, W65I, D66T, and K68T) were termed Lipr27RCL-K64N, Lipr27RCLW65I, Lipr27RCL- D66T, and Lipr27RCL-K68T for the purposes of this study. These lipases had the same molecular mass $(39.50 \mathrm{kDa})$ as did Lipr27RCL when analyzed via SDS-PAGE (Figure 1). Following purification, we found the specific activities of Lipr27RCL, Lipr27RCL-K64N, Lipr27RCL-W65I, Lipr27RCLD66T, and Lipr27RCL-K68T to be 2218.52, 2379.71, 1961.14, 2313.66, and $2449.40 \mathrm{U} / \mathrm{mg}$, respectively.

We next assessed the changes in lipase thermostability as a result of this mutagenic campaign by assessing residual activity of these four Lipr27RCL enzymes and the WT isoform following a 90 min incubation at a range of temperatures. We found that all five of these enzymes functioned best at $40^{\circ} \mathrm{C}$ (Figure 3). Both Lipr27RCL-K64N and Lipr27RCL-K68T remained stable at $50^{\circ} \mathrm{C}$, with residual activities of 66.12 and $76.44 \%$ after a $2 \mathrm{~h}$ incubation at this temperature, respectively. In contrast, the residual activities of the other lipase isoforms declines substantially under these conditions. Lipr27RCL and Lipr27RCLD66T had half-lives of $65 \mathrm{~min}$ under these conditions, with 28.24 and $28.60 \%$ residual activities, respectively, after a $2 \mathrm{~h}$ treatment. Lipr27RCL-W65I had a half-life of just $40 \mathrm{~min}$ and a residual activity of $10.09 \%$ following this $2 \mathrm{~h}$ treatment (Figure 4A). At temperatures above $50^{\circ} \mathrm{C}$, Lipr27RCL-K64N and Lipr27RCL-K68T were also more stable than the other tested enzymes. Lipr27RCL-K64N retained $49.23 \%$ activity following $1 \mathrm{~h}$ at $60^{\circ} \mathrm{C}$, which was approximately the halflife of this enzyme, and it retained $29.84 \%$ activity following 


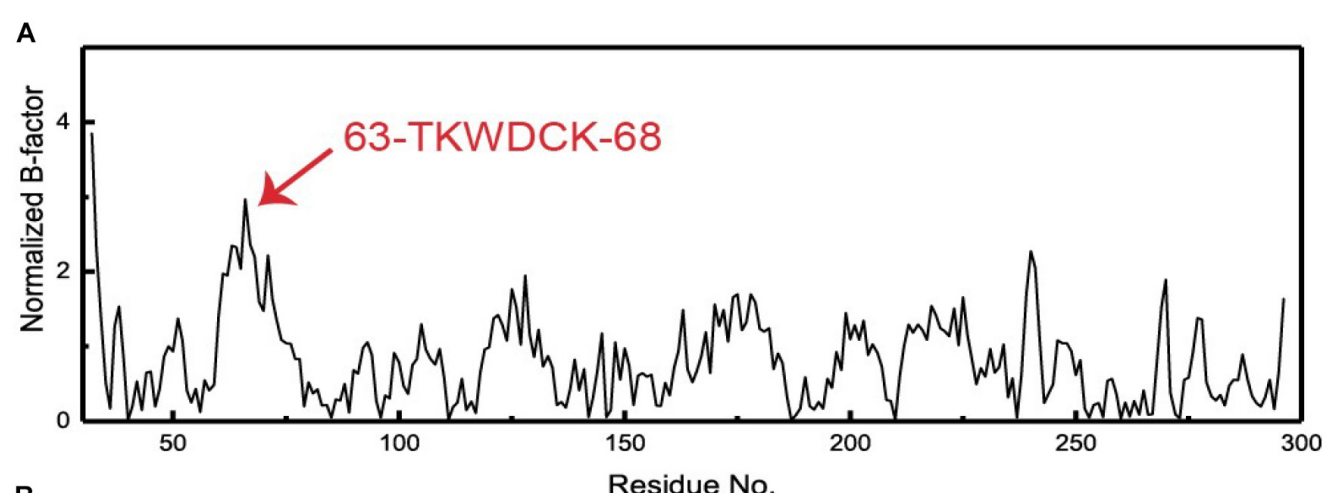

B

Residue No.

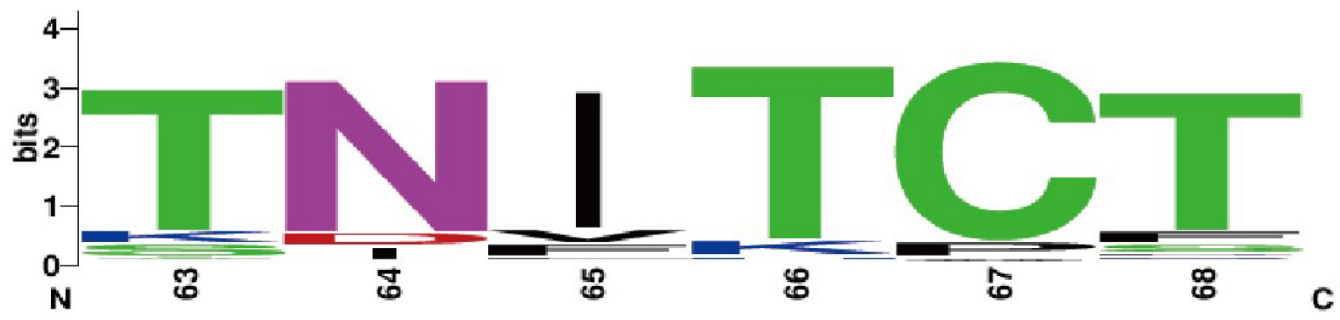

FIGURE 2 | (A) Normalized B-factor Lipr27RCL and (B) multiple sequence alignment based on 92 lipase sequences from different species of thermophilic fungi.

a $90 \mathrm{~min}$ incubation at this temperature. Lipr27RCL-K68T retained $51.44 \%$ activity after a $75 \mathrm{~min}$ incubation at $60^{\circ} \mathrm{C}$, which was close to its half-life, and it retained $41.47 \%$ activity following a $90 \mathrm{~min}$ incubation at this temperature. In contrast, Lipr27RCL retained $53.28 \%$ activity after only $25 \mathrm{~min}$ at $60^{\circ} \mathrm{C}$, with just $5.34 \%$ activity being retained following $90 \mathrm{~min}$ at this temperature. Similarly, Lipr27RCL-D66T retained $48.90 \%$ activity after $20 \mathrm{~min}$ at $60^{\circ} \mathrm{C}$, with $4.44 \%$ activity having been retained after $90 \mathrm{~min}$ at this temperature. After just $10 \mathrm{~min}$ at $60^{\circ} \mathrm{C}$, Lipr27RCL-W65I retained just $48.9 \%$ activity, with no activity at all remaining after $75 \mathrm{~min}$ at this temperature (Figure 4B). Relative to the WT lipase isoform, Lipr27RCLK64N and Lipr27RCL-K68T exhibited residual activities which were increased by $37.88 \%$ and $48.20 \%$, respectively, after $120 \mathrm{~min}$ at $50^{\circ} \mathrm{C}$, and their respective half-lives had increased by 2.4 and 3.0 -fold at $60^{\circ} \mathrm{C}$ for $90 \mathrm{~min}$. Lipr27RCL-D66T performed nearly identically to WT Lipr27RCL, whereas Lipr27RCLW65I had poorer thermostability than did WT Lipr27RCL. These findings thus suggested that two of the tested amino acid substitutions (K64N and K68T) were advantageous for Lipr27RCL thermostability.

\section{Kinetic Analysis of Mutant Lipr27RCL Isoforms}

We assessed the kinetic parameters of these five lipase isoforms at a $\mathrm{pH}$ of 9.0 at $37^{\circ} \mathrm{C}$. The $p$-NP method was used to monitor these reactions, with purified proteins being combined with a range of 4-PNN concentrations (0.078125-10 mM). Kinetic measurements revealed that the apparent Michaelis constant $\left(K_{m}\right)$ values for Lipr27RCL, Lipr27RCL-K64N, Lipr27RCL-W65I, Lipr27RCL-D66T, and

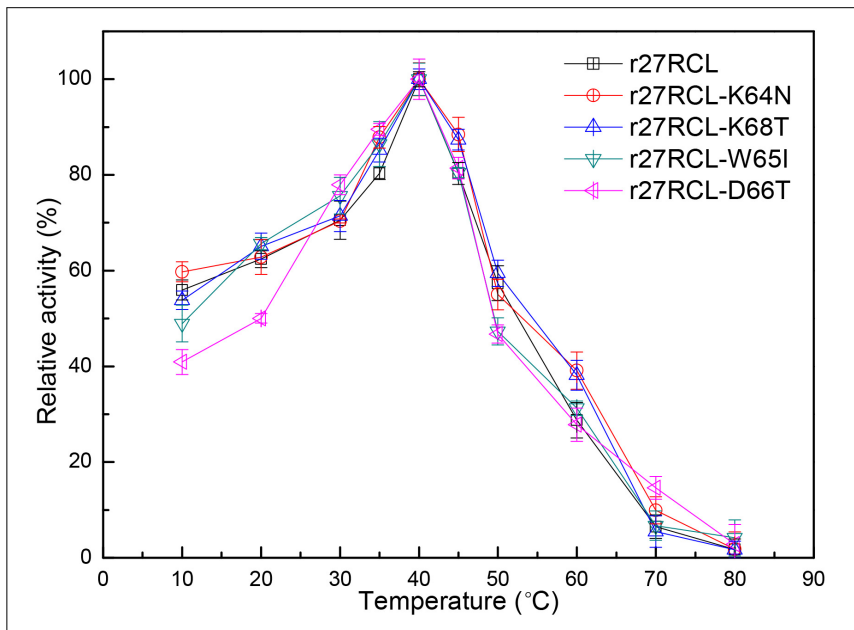

FIGURE 3 | The impact of temperature on recombinant Lipr27RCL, Lipr27RCL-K64N, Lipr27RCL-W65, Lipr27RCL-D66T, and Lipr27RCL-K68T.

Lipr27RCL-K68T were $0.36,0.29,0.45,0.38$, and $0.29 \mathrm{mM}$, respectively (Table 2 ). The smaller $K_{m}$ values for Lipr27RCLK64N and Lipr27RCL-K68T were indicative of the increased kinetic efficiency of these enzymes relative to WT Lipr27RCL. The catalytic efficiency $\left(k_{\text {cat }} / K_{m}\right)$ of Lipr27RCL-K64N and Lipr27RCL-K68T was also increased (1.22-fold and 1.24-fold, respectively). The $K_{m}$ and $k_{\text {cat }} / K_{m}$ values for Lipr27RCLD66T were similar to those for WT Lipr27RCL. However, the $K_{m}$ of Lipr27RCL-W65I was 1.25-fold that of Lipr27RCL, with a clear decline in $k_{\text {cat }} / K_{m}$ (Table 2). These kinetic analyses thus revealed that the K64N and K68T substitutions, 

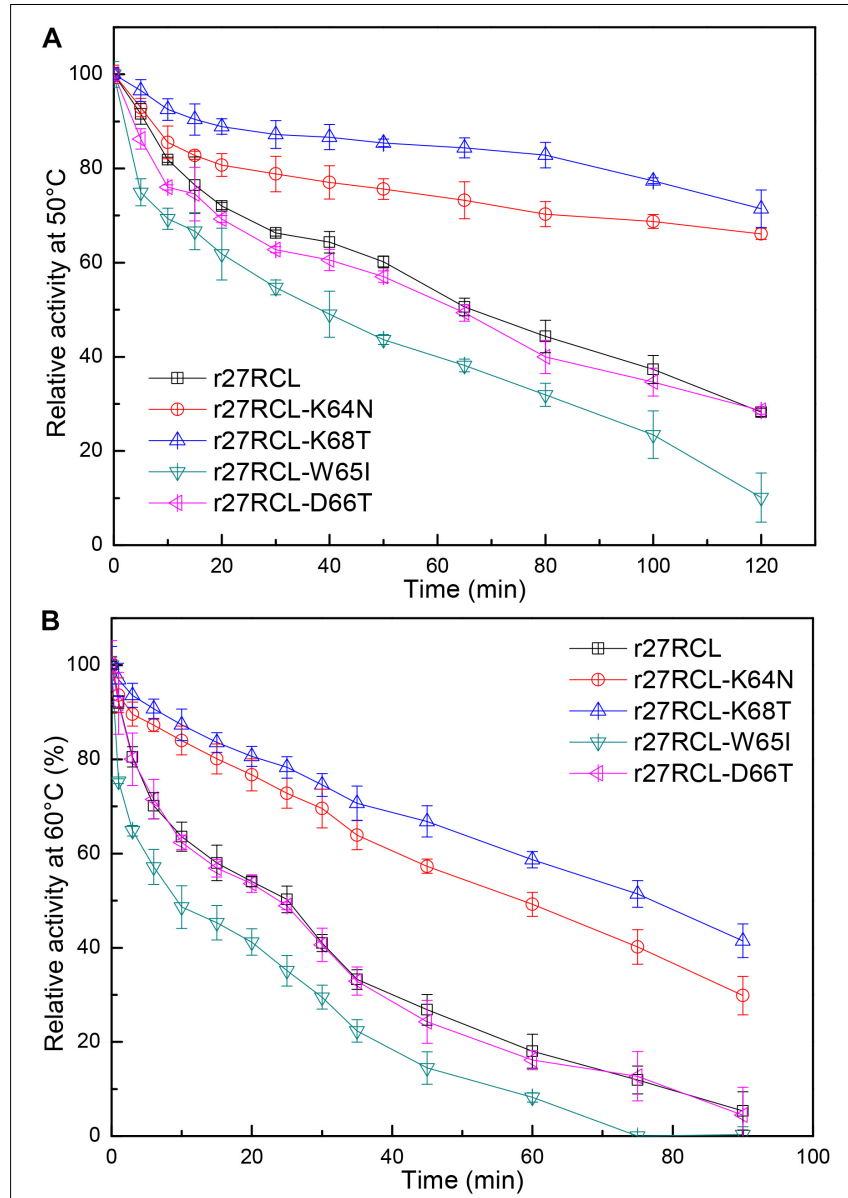

FIGURE 4 | The thermostability of Lipr27RCL, Lipr27RCL-K64N, Lipr27RCL-W65, Lipr27RCL-D66T, and Lipr27RCL-K68T at 50 $\mathrm{C}$ (A) and $60^{\circ} \mathrm{C}(\mathbf{B})$.

although primarily intended to improve thermostability, also enhanced the catalytic efficiency and substrate binding of these lipases.

\section{Circular Dichroism (CD) Spectroscopy}

We selected Lipr27RCL-K64N and Lipr27RCL-K68T with improved thermal stability using $\mathrm{CD}$ spectroscopy to compare with Lipr27RCL to prove whether the single mutation caused

TABLE 2 | Kinetics of Lipr27RCL, Lipr27RCL-K64N, Lipr27RCL-W65, Lipr27RCL-D66T, and Lipr27RCL-K68T.

\begin{tabular}{|c|c|c|c|c|}
\hline \multirow[t]{2}{*}{ Enzymes } & $V_{\max }$ & $K_{m}$ & $k_{\text {cat }}$ & $k_{c a t} / K_{m}$ \\
\hline & (U/mg) & (mM) & $(/ \mathrm{s})$ & $(/ \mathrm{s} / \mathrm{mM})$ \\
\hline Lipr27RCL & $714.29 \pm 23.57$ & $0.36 \pm 0.06$ & $188.10 \pm 18.21$ & 522.50 \\
\hline Lipr27RCL-K64N & $588.24 \pm 19.63$ & $0.29 \pm 0.09$ & $184.86 \pm 18.46$ & 637.45 \\
\hline Lipr27RCL-W65 & $500.00 \pm 23.02$ & $0.45 \pm 0.04$ & $131.50 \pm 19.38$ & 292.22 \\
\hline Lipr27RCL-D66T & $709.00 \pm 19.03$ & $0.38 \pm 0.05$ & $186.66 \pm 16.28$ & 491.21 \\
\hline Lipr27RCL-K68T & $714.29 \pm 20.21$ & $0.29 \pm 0.06$ & $187.95 \pm 13.65$ & 648.10 \\
\hline
\end{tabular}

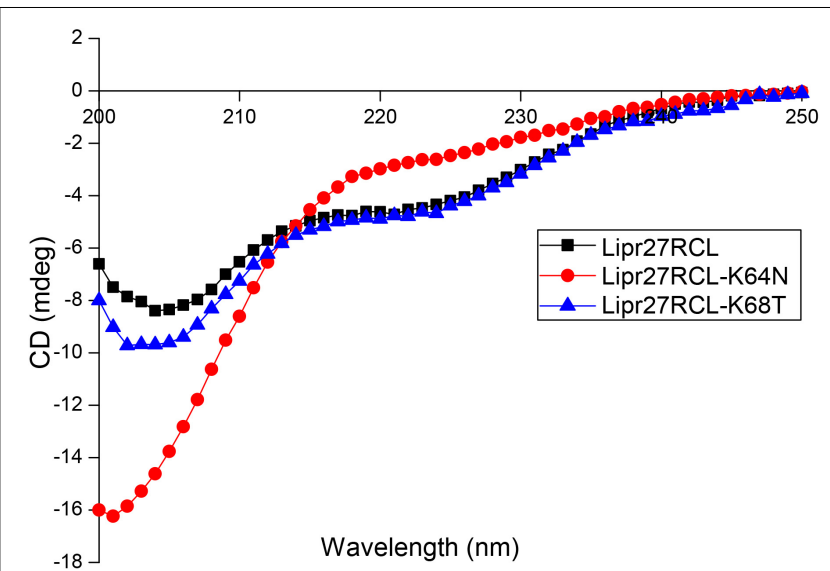

FIGURE 5 | Circular dichroism spectra (CD) of Lipr27RCL, Lipr27RCL-K64N, and Lipr27RCL-K68T.

changes in the secondary structure of Lipr27RCL. CD technology can give information under the protein conformation in solution through the dependence of the optical activity of a peptide chain, with almost no side chain interference (except aromatic amino acids) (Nascimento et al., 2019). The CD spectra of the three lipase isoforms (Lipr27RCL, Lipr27RCL-K64N, and Lipr27RCL-K68T) are shown in Figure 5. In addition, to investigate the thermal denaturation and renaturation of these three isoforms, we heat-treated them at $65^{\circ} \mathrm{C}$ for $5 \mathrm{~min}$ and placed them on ice, and then performed $\mathrm{CD}$ spectrum detection. At the same time, the renaturation samples were heat-treated at $65^{\circ} \mathrm{C}$ and placed on ice, and then recovered at $4^{\circ} \mathrm{C}$ for 2 days for $\mathrm{CD}$ spectrum scan (Supplementary Figure S1).

The CD spectra showed that compared with Lipr27RCL, the ellipticities of the $\alpha$-helix and $\beta$-sheet of the Liper27RCLK64N isoform changed obviously, while the ellipticity of $\alpha$-helix of Lipr27RCL-K68T isoform had minor changes with almost no changes of $\beta$-sheet (Figure 5). These suggested that mutations at these two single sites might cause changes in the secondary structure of the Lipr27RCL isoform. In addition, the CD spectra of Liper27RCL-K64N and Liper27RCL-K68T were also different, indicating that there were some differences in the thermal stability of the two mutants. Moreover, the CD spectra of the three thermal denaturation isoforms showed that only the ellipticities of $\beta$-sheet of the Lipr27RCL-K64N and Lipr27RCL-K68T isoforms changed after heat treatment, while the ellipticities of $\alpha$-helix and $\beta$-sheet of Lipr27RCL both changed (Supplementary Figure S1). These indicated that the thermal stability of Lipr27RCL-K64N and Lipr27RCL-K68T isoforms might be improved. However, the $\mathrm{CD}$ spectra of the three isoforms of thermal denaturation and renaturation showed that the ellipticities of the $\alpha$-helix and $\beta$-sheet of these isoforms had little change before and after thermal denaturation and renaturation (Supplementary Figure S1). These indicated that it was difficult for them to recover enzyme activity after denaturation. 


\section{Enhanced Thermal Tolerance of the Single Mutants Explored by MD Simulations}

To fully understand the basis of enhanced thermostability in these recombinant lipases, MD simulations for Lipr27RCL and two single mutants (Lipr27RCL-K64N and Lipr27RCL-K68T) with improved thermostability were conducted at $60^{\circ} \mathrm{C}$. To investigate the improved thermostability of mutants, novel interactions formed with the mutational sites were monitored during the simulations. It is discovered hydrogen bonding interactions between N64..S58 in Lipr27RCL-K64N was stronger than that in K64..S58 Lipr27RCL. Specifically, the hydrogen bond forming probability between K/N64. .S58 were 70.6\% and $62.5 \%$ in Lipr27RCL-K64N and Lipr27RCL, respectively (Figures 6A,B). Additionally, comparing the root mean square fluctuation (RMSF) values which reflect the flexibility of the mutated residues in the three lipases, we found that RMSF value of the mutated residue N64 in Lipr27RCL-K64N was smaller than that of K64 in Lipr27RCL, indicating improved stability of the mutated residue (Table 3). The side chain of N64 is smaller than that of K64, and as a result N64 exhibited increased local stability owing to its mild floating amplitude, and this coupled with the retention of normal hydrogen bond interactions led to the enhanced thermostability of Lipr27RCL-K64N.

Similarly, RMSF value of the mutated residue T68 in Lipr27RCL-K68T was smaller than that of K68 in Lipr27RCL, and the smaller RMSF value of T68 indicate the mutated site was relative stable during simulation (Table 3). T68 in Lipr27RCL$\mathrm{K} 68 \mathrm{~T}$ also had a shorter side chain relative to that of K68, and the

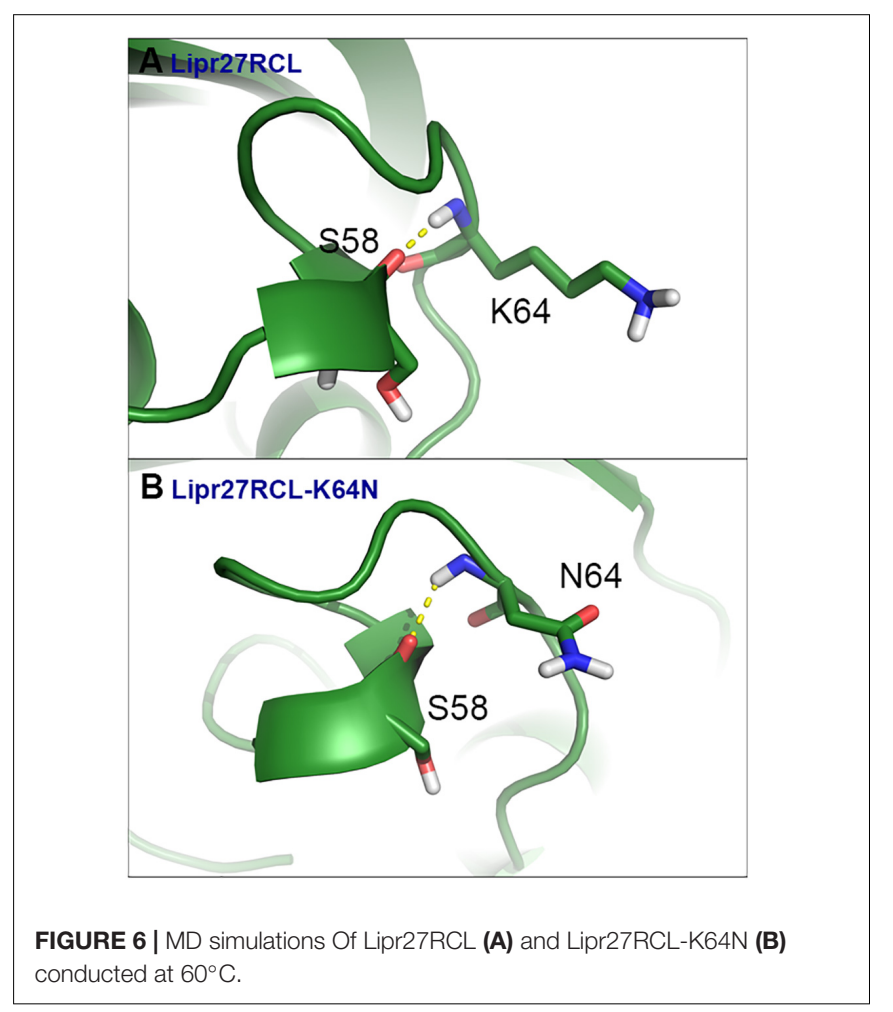

TABLE 3 | RMSF for mutated residues over the whole simulation in Lipr27RCL and two mutants.

\begin{tabular}{lcc}
\hline Enzymes & K/N64 (nm) & K/T68 (nm) \\
\hline Lipr27RCL & 0.1576 & 0.1995 \\
Lipr27RCL-K64N & 0.1187 & 0.1786 \\
Lipr27RCL-K68T & 0.1636 & 0.1478 \\
\hline
\end{tabular}

$\alpha$-helix where T68 located was thus more stable than that of the wild type enzyme.

\section{DISCUSSION}

The development of thermostable lipases has clear and practical potential to meet with industrial demands. Furthermore, generating such stable lipases may simultaneously improve their kinetic efficiency (Rogers and Bommarius, 2010; Han et al., 2017). In the present study, we utilized a B-factor comparison and multisequence alignment strategy to guide our efforts to design and produce thermostable lipase mutants. The resultant Lipr27RCLK64N and Lipr27RCL-K68T proteins, which were designed via site-directed mutagenesis, exhibited both higher thermostability and superior catalytic efficiency.

Normalized B-factor analysis of Lipr27RCL was the key step in the design of thermostable recombinant isoforms of this enzyme, given that B-factors offer insight into protein fluctuations, which are indicative of atom rigidity relative to their corresponding positions (Ringe and Petsko, 1986), and previous work has shown that improved thermostability depends upon achieving a higher degree of rigidity (Podar and Reysenbach, 2006). As such, we analyzed normalized B-factor values from Lipr27RCL, and aligned this sequence with those of other thermophilic lipases using sequences from NCBI. We were thereby able to identify residues with pronounced degrees of flexibility, and we then constructed recombinant mutant lipases (Lipr27RCL-K64N, Lipr27RCL-W65I, Lipr27RCL-D66T, and Lipr27RCL-K68T) in which these flexible residues had been mutated. In subsequent thermostability and kinetic analyses, we found that Lipr27RCLK64N and Lipr27RCL-K68T exhibited superior thermostability, substrate affinity, and catalytic efficiency. As such, these results confirm that B-factor analysis can facilitate efforts to selectively improve lipase thermostability profiles.

Based on sequence and structural analyses, we found further evidence suggesting that Lipr27RCL-K64N and Lipr27RCL-K68T may exhibit greater thermostability relative to other untested lipase isoforms, leading us to focus our in depth characterization efforts on these isoforms. We had also constructed a quadruple mutant lipase (K64N, W65I, D66T, and K68T) for this study, but found its thermostability to be $40 \%$ lower than the WT Lipr27RCL in a matter of minutes. This is likely at least in part due to the loss of hydrogen bond network interactions introduced by the I65 mutation, thereby adversely impacting the formation of hydrogen bond networks and the $\alpha$-helix around N64 and T68. This loss of hydrogen bond networks in turn reduced the regional thermostability, markedly decreasing the thermostability of this quadruple mutant lipase. As such, other lipase isoforms 
containing the I65 mutation will similarly not display improved thermostability owing to this loss of hydrogen bond network interactions. Similarly, lipase isoforms bearing T66 mutations are likely to exhibit limited thermostability due to the lack of stable interactions between this residue and other surrounding residues. As such, the characterization of lipase isoforms containing mutated 165 or T66 residues was not tested in this work. However, the double mutant lipase (Lipr27RCL-K64N/K68T) exhibited cumulative thermostability of Lipr27RCL-K64N and Lipr27RCLK68T (Supplementary Figure S2), indicating that the role of the two mutation sites may be aggregate.

In summary, we found that we were able to produce novel mutant lipases (Lipr27RCL-K64N and Lipr27RCL-K68T) with improved thermostability through a combination of $\mathrm{B}$ factor analysis and site-directed mutagenesis, and these resultant enzymes represent attractive candidates for use in industrial applications.

\section{DATA AVAILABILITY STATEMENT}

The raw data supporting the conclusions of this article will be made available by the authors, without undue reservation, to any qualified researcher.

\section{AUTHOR CONTRIBUTIONS}

CZ performed the major experiments containing enzyme production, purification, and CD analysis. ZJ and MT carried out the experiments containing site-directed mutagenesis and

\section{REFERENCES}

Arnold, K., Bordoli, L., Kopp, J., and Schwede, T. (2006). The SWISSMODEL workspace: a web-based environment for protein structure homology modelling. Bioinformatics 22, 195-201. doi: 10.1093/bioinformatics/bti770

Bommarius, A. S., and Paye, M. F. (2013). Stabilizing biocatalysts. Chem. Soc. Rev. 42, 6534-6565. doi: 10.1039/C3CS60137D

Ferreira-Dias, S., Sandoval, G., Plou, F., and Valero, F. (2013). The potential use of lipases in the production of fatty acid derivatives for the food and nutraceutical industries. Electron. J. Biotechn. 16, 1-38. doi: 10.2225/vol16-issue3-fulltext-5

Goomber, S., Kumar, R., Singh, R., Mishra, N., and Kaur, J. (2016). Point mutation Gln121-Arg increased temperature optima of Bacillus lipase (1.4 subfamily) by fifteen degrees. Int. J. Biol. Macromol. 88, 507-514. doi: 10.1016/j.ijbiomac. 2016. 04.022

Gupta, R., Kumari, A., Syal, P., and Singh, Y. (2015). Molecular and functional diversity of yeast and fungal lipases: their role in biotechnology and cellular physiology. Prog. Lipid. Res. 57, 40-54. doi: 10.1016/j.plipres.2014.12.001

Han, N., Miao, H., Ding, J., Li, J., Mu, Y., Zhou, J., et al. (2017). Improving the thermostability of a fungal GH11 xylanase via site-directed mutagenesis guided by sequence and structural analysis. Biotechnol. Biofuels 10:133. doi: 10.1186/ s13068-017-0824-y

Hess, B., Kutzner, C., Van Der Spoel, D., and Lindahl, E. (2008). GROMACS 4: algorithms for highly efficient, load-balanced, and scalable molecular simulation. J. Chem. Theory Comput. 4, 435-447. doi: 10.1021/ct700 $301 \mathrm{q}$

Hornak, V., Abel, R., Okur, A., Strockbine, B., Roitberg, A., and Simmerling, C. (2006). Comparison of multiple Amber force fields and development of improved protein backbone parameters. Proteins 65, 712-725. doi: 10.1002/ prot. 21123 characterization of lipases. LW, WQ, JH, and $\mathrm{ZH}$ helped with protein purification. $\mathrm{NH}$ analyzed the B-factor and $\mathrm{MD}$ simulation. QW and YM prepared experimental materials. $\mathrm{BX}, \mathrm{JD}$, and RZ dealt with the problems encountered in the experiment and coordinated the study. $\mathrm{CZ}$ wrote the manuscript. $\mathrm{ZH}$ and $\mathrm{NH}$ revised this manuscript. All authors read and approved the final manuscript.

\section{FUNDING}

This work was supported by the National Key Research and Development Program of China (Grant Number 2017YFB0308401), the National Natural Science Foundation of China (Grant Numbers 31660240, 31660304, and 31960131), and the Yunling Scholar Fund and Yunling Technical Leader Fund.

\section{SUPPLEMENTARY MATERIAL}

The Supplementary Material for this article can be found online at: https://www.frontiersin.org/articles/10.3389/fmicb. 2020.00346/full\#supplementary-material

FIGURE S1 | Circular dichroism spectra (CD) of Lipr27RCL (A), Lipr27RCL-K64N (B), and Lipr27RCL-K68T (C) under the condition of thermal denaturation at $65^{\circ} \mathrm{C}$ for $5 \mathrm{~min}$ and renaturation at $4^{\circ} \mathrm{C}$ for $2 \mathrm{~d}$, respectively.

FIGURE S2 | The thermostability of Lipr27RCL, Lipr27RCL-K64N, Lipr27RCL-K68T, and Lipr27RCL-K64N/ K68T at $60^{\circ} \mathrm{C}$.

FIGURE S3 | The original image of Figure 1.

Jorgensen, W. L., Chandrasekhar, J., Madura, J. D., Impey, R. W., and Klein, M. L. (1983). Comparison of simple potential functions for simulating liquid water. J. Chem. Phys. 79, 926-935. doi: 10.1063/1.445869

Li, G., Fang, X., Su, F., Chen, Y., Xu, L., and Yan, Y. (2018). Enhancing the thermostability of Rhizomucor miehei lipase with a limited screening library by rational-design point mutations and disulfide bonds. Appl. Environ. Microbiol. 84:e2129-17. doi: 10.1128/AEM.02129-17

Li, Z., Li, X., Wang, Y., Wang, Y., Wang, F., and Jiang, J. (2011). Expression and characterization of recombinant Rhizopus oryzae lipase for enzymatic biodiesel production. Bioresour. Technol. 102, 9810-9813. doi: 10.1016/j.biortech.2011. 07.070

Lineweaver, H., and Burk, D. (1934). The determination of enzyme dissociation constants. J. Am. Chem. Soc. 56, 658-666. doi: 10.1021/ja01318a036

Liu, Y. H., Liu, H., Huang, L., Gui, S., Zheng, D., Jia, L. B., et al. (2017). Improvement in thermostability of an alkaline lipase I from Penicillium cyclopium by directed evolution. RSC Adv. 7, 38538-38548. doi: 10.1039/ C7RA06307E

Naik, S., Basu, A., Saikia, R., Madan, B., Paul, P., Chaterjee, R., et al. (2010). Lipases for use in industrial biocatalysis: specificity of selected structural groups of lipases. J. Mol. Catal. B Enzym. 65, 18-23. doi: 10.1016/j.molcatb.2010.01.002

Nascimento, P. A. M., Pereira, J. F. B., and De Carvalho Santos-Ebinuma, V. (2019). Insights into the effect of imidazolium-based ionic liquids on chemical structure and hydrolytic activity of microbial lipase. Bioprocess. Biosyst. Eng. 42, 1235-1246. doi: 10.1007/s00449-019-02121-w

Pandey, A., Benjamin, S., Soccol, C. R., Nigam, P., Krieger, N., and Soccol, V. T. (1999). The realm of microbial lipases in biotechnology. Biotechnol. Appl. Biochem. 29, 119-131. doi: 10.1111/j.1470-8744.1999.tb00541.x

Persson, M., Costes, D., Wehtje, E., Adlercreutz, P. J. E., and Technology, M. (2002). Effects of solvent, water activity and temperature on lipase and 
hydroxynitrile lyase enantioselectivity. Enzyme Microb. Technol. 30, 916-923. doi: 10.1016/S0141-0229(02)00033-9

Podar, M., and Reysenbach, A. L. (2006). New opportunities revealed by biotechnological explorations of extremophiles. Curr. Opin. Biotechnol. 17, 250-255. doi: 10.1016/j.copbio.2006.05.002

Ringe, D., and Petsko, G. A. (1986). Study of protein dynamics by X-ray diffraction. Method Enzymol. 131, 389-433. doi: 10.1016/0076-6879(86)31050-4

Rogers, T. A., and Bommarius, A. S. (2010). Utilizing simple biochemical measurements to predict lifetime output of biocatalysts in continuous isothermal processes. Chem. Eng. Sci. 65, 2118-2124. doi: 10.1016/j.ces.2009. 12.005

Sha, C., Yu, X. W., Zhang, M., and Xu, Y. (2013). Efficient secretion of lipase r27RCL in Pichia pastoris by enhancing the disulfide bond formation pathway in the endoplasmic reticulum. J. Ind. Microbiol. Biotechnol. 40, 1241-1249. doi: 10.1007/s10295-013-1328-9

Stepankova, V., Bidmanova, S., Koudelakova, T., Prokop, Z., Chaloupkova, R., and Damborsky, J. (2013). Strategies for stabilization of enzymes in organic solvents. ACS Catal. 3, 2823-2836. doi: 10.1021/cs400684x

Su, F., Li, G. L., Zhang, H. J., and Yan, Y. J. (2014). Enhanced performance of Rhizopus oryzae lipase immobilized on hydrophobic carriers and its application in biorefinery of rapeseed oil deodorizer distillate. Bioenerg. Res. 7, 935-945. doi: 10.1007/s12155-014-9415-y

Suen, W. C., Zhang, N. Y., Xiao, L., Madison, V., and Zaks, A. (2004). Improved activity and thermostability of Candida antarctica lipase B by DNA family shuffling. Protein Eng. Des. Sel. 17, 133-140. doi: 10.1093/protein/gzh017

Xie, Y., An, J., Yang, G., Wu, G., Zhang, Y., Cui, L., et al. (2014). Enhanced enzyme kinetic stability by increasing rigidity within the active site. J. Biol. Chem. 289, 7994-8006. doi: 10.1074/jbc.M113.536045
Yu, X. W., Tan, N. J., Xiao, R., and Xu, Y. (2012a). Engineering a disulfide bond in the lid hinge region of Rhizopus chinensis lipase: increased thermostability and altered acyl chain length specificity. PLoS One 7:e46388. doi: 10.1371/journal. pone. 0046388

Yu, X. W., Wang, R., Zhang, M., Xu, Y., and Xiao, R. (2012b). Enhanced thermostability of a Rhizopus chinensis lipase by in vivo recombination in Pichia pastoris. Microb. Cell. Fact. 11:102. doi: 10.1186/1475-2859-11-102

Yu, X. W., Zhu, S. S., Xiao, R., and Xu, Y. (2014). Conversion of a Rhizopus chinensis lipase into an esterase by lid swapping. J. Lipid. Res. 55, 1044-1051. doi: 10.1194/jlr.M043950

Yuan, Z., Zhao, J., and Wang, Z. X. (2003). Flexibility analysis of enzyme active sites by crystallographic temperature factors. Protein Eng. 16, 109-114. doi: $10.1093 /$ proeng/gzg014

Conflict of Interest: LW, WQ, JH, and ZZ were employed by Yunnan Walvax Biotechnology Co., Ltd.

The remaining authors declare that the research was conducted in the absence of any commercial or financial relationships that could be construed as a potential conflict of interest.

Copyright (c) 2020 Jiang, Zhang, Tang, Xu, Wang, Qian, He, Zhao, Wu, Mu, Ding, Zhang, Huang and Han. This is an open-access article distributed under the terms of the Creative Commons Attribution License (CC BY). The use, distribution or reproduction in other forums is permitted, provided the original author(s) and the copyright owner(s) are credited and that the original publication in this journal is cited, in accordance with accepted academic practice. No use, distribution or reproduction is permitted which does not comply with these terms. 